\title{
Hyperbaric Oxygen Suppressed Tumor Progression through Improvement of Tumor Hypoxia and Induction of Tumor Apoptosis in Lung Cancer
}

Shao-Yuan Chen ( $\nabla$ 1sychen@yahoo.com.tw)

Cardinal Tien Hospital

Koichi Tsuneyama

University of Tokushima

Mao-Hsiung Yen

National Defense Medical Center

Jiunn-Tay Lee

National Defense Medical Center

Jiun-Liang Chen

Chang Gung University

Shih-Ming Huang

National Defense Medical Center

\section{Research Article}

Keywords: Tumor cells, hyperbaric oxygen treatment (HBOT), lung cancer

Posted Date: January 8th, 2021

DOl: https://doi.org/10.21203/rs.3.rs-137802/v1

License: (1) This work is licensed under a Creative Commons Attribution 4.0 International License.

Read Full License 


\section{Abstract}

Tumor cells have long term been recognized as a relative contraindication to hyperbaric oxygen treatment (HBOT) since HBOT might enhance progressive cancer growth. However, in an oxygen deficit condition, tumor cells are more progressive and have the potentials to be metastatic. HBOT increasing in oxygen partial pressure may benefit tumor suppression. In this study, we investigated the effects of HBOT on solid tumors, such as lung cancer. Non-small cell human lung carcinoma A549-cell-transferred severe combined immunodeficiency mice (SCID) mice were selected as an in vivo model to detect the potential mechanism of HBOT in lung tumors. HBOT not only improved tumor hypoxia but also suppressed tumor growth in murine xenograft tumor models. In vitro, HBOT suppressed the growth of A549 cells in a timedependent manner and immediately downregulated the expression of p53 protein after HBOT in A549 cells. Our results demonstrated that HBOT improved tissue vasculogenesis, tumor hypoxia and potentially target apoptosis to lung cancer cells in murine xenograft tumor models. HBOT will merit further cancer therapy as an adjuvant treatment for lung cancer.

\section{Introduction}

Tumor hypoxia has been a major problem for cancer therapy [1, 2]. Hypoxia can increase tumor resistance to chemotherapy, radiation and lead to malignant progression and even metastasis $[3,4]$. Hypoxia or some signaling alterations of the hypoxic cascades could enhance the intact neovascularization, which facilitates tumor metastasis $[5,6]$.

Hyperbaric Oxygen Treatment (HBOT) is a medical treatment using $100 \%$ oxygen administered at a greater than normal atmospheric pressure to patients. Since cellular and vascular proliferation is promoted by HBOT in an ischemic wound $[7,8]$, practitioners of hyperbaric medicine have concerned about the effect of HBOT on cancer growth [9]. However, more and more evidence suggests a neutral effect of HBOT on malignancy [10-12]. HBOT working as an adjuvant to promote cellular and vascular proliferation might have the same effect in a tumor. However, the nature physiology between tumor growth and wound healing is very different by the way of cancer growth, transformation and metastases. Such differences will result in different impacts on HBOT. A major difference pointed out by Growther et al [13] suggested macrophages are the major healing factors in angiogenesis, while tumor macrophages contributed as part as an angiogenesis factor in a tumor environment $[14,15]$. The optimal oxygen tensions of 30 to $40 \mathrm{mmHg}$ increased by HBOT can stimulate collagen synthesis and hydroxylation in wounds, but is not working to promote cell proliferation in cancer.

Our previous experiments demonstrated that exposure to HBO attenuated the severity of disease progression in autoimmune NZB/W F1 mice [16]; proposing targeted apoptosis to hyper-proliferating lymphocytes. Moreover, cells of hematopoietic origin have a lower threshold to oxidative stress induced by HBOT and HBO-induced apoptosis of hematopoietic derived cancer cells was through the intracellular accumulation of $\mathrm{H}_{2} \mathrm{O}_{2}$ and $\mathrm{O}_{2} \cdot-$ as well as the involvement of phosphorylation of p38 MAPK [17]. 
Lung cancer is responsible for more than one million deaths annually and is diagnosed with the most popular cancer in the world. The main types of lung cancer are small-cell lung cancer (SCLC), and nonsmall-cell lung cancer (NSCLC). Several studies so far have provided direct beneficial shreds of evidence to show the effective combined therapy with HBOT and chemotherapy for cancer. The reasons for the successfully combined therapy are the good vascularization of hypoxic tumor cells after HBOT. Hypoxic tumor cells are much more progressive and have the potentials to be metastatic. Moreover, tumor cells under hypoxic situations are more resistant to chemotherapy. In several clinical studies, the combination of HBOT and chemotherapy demonstrated a beneficial value of increased survival rate and the side effects did not increase by combining HBOT with chemotherapy [18-20].

Our study aimed to establish human lung carcinoma A549 tumor transferred severe combined immunodeficiency mice (SCID) mice to investigate the potential role of HBOT in cancer therapy. NSCLC A549-transferred SCID mice were selected to detect the long-term beneficial effects of HBOT in cancer therapy without any combined treatment in the manner of 2.5 ATA/90 min/day in 2 weeks after 45 days of tumor transfer. Tumor hypoxia detected by Hypoxyprobe ${ }^{T M}-1$ was examined and the effect of HBOT on tumor growth was demonstrated after 14 and 28 days of HBOT. Further, the direct effects of HBOT on cell base, including cell viability and related protein expressions (such as p53 and HIF-1a) were detected in A549 cells. Hence, this study will be the first experiment to provide both in vivo and in vitro responses of tumor cells to HBOT and propose the underlined tumor microenvironment for further lung cancer treatment

\section{Results}

\section{HBOT suppressed tumor growth.}

On the observation that HBOT could decrease the proliferation rate of A549 cells, we established A549 tumor transferred-SCID mice xenograft model for the potential use of HBOT (2.5 ATA, $90 \mathrm{~min}$ ) in cancer therapy. A549-tumor transferred SCID mice were either non-exposed or exposed to HBO for 2.5 ATA 90 min 5 times in a week beginning at the $45^{\text {th }}$ date after tumor injection and were monitored for the tumor volume twice in a week. The tumor volume of human lung carcinoma A549 cells was increased as a linear curve after 20 days of transfer for both control and HBO-treated mice(Figure $1 \mathrm{~A}$ ). Only the mice with tumor volume larger than $500 \mathrm{~mm}^{3}$ were selected to HBOT. Our data showed that HBOT effectively suppressed tumor growth after 14 days $\left({ }^{* *} \mathrm{P}<0.01\right)$ and 28 days $\left({ }^{* *} \mathrm{P}<0.01\right)$ of tumor transfer (Figure $1 \mathrm{~A}$, B) compared with control mice.

\section{HBOT improved tumor hypoxia.}

Although tumor sections from A549-transferred SCID mice were showing the regular shapes after 45 days of tumor transfer by hematoxylin\& eosin ( $\mathrm{H} \& \mathrm{E})$ staining, significant tumor hypoxia was measured by 
Hypoxyprobe ${ }^{T M}-1$ probe (Figure $\left.2 A\right)$. HBOT significantly improved tumor hypoxia after 14 days $\left({ }^{*} \mathrm{P}<0.05\right)$ and 28 days $\left({ }^{*} \mathrm{P}<0.05\right)$ of $\mathrm{HBO}$ therapy (Figure $2 \mathrm{~B}$, Table 1$)$ by semi-quantitative analysis.

\section{HBOT induced apoptosis in A549 tumor transferred SCID mice.}

Histopathological findings of tumor lesion were quite similar at the $0^{\text {th }}$ date after $\mathrm{HBO}$ exposure between control and HBOT groups. The area of the necrotic field is not observed at the $0^{\text {th }}$ date after HBOT; however necrotic field was more prominent at the $14^{\text {th }}$ and $28^{\text {th }}$ date after HBOT. Ghost-like shadow of cells was recognized in the necrotic field as "coagulation necrosis". Tumor sections of HBOT-14 ${ }^{\text {th }}$ and $28^{\text {th }}$ date showed zonal inflammatory cells and degenerated cells with nuclear debris were aggregated in the border of coagulation necrosis (Figure 3A). Immunostaining of cleaved-caspase-3 was demonstrated in HBO-treated mice after 14 days of HBOT (Figure 3B) compared with control mice (Figure 3C).

\section{CD31 was significantly increased after HBOT.}

Platelet endothelial cell adhesion molecule (PECAM-1/CD31), is a ligand for CD38 and plays a role in angiogenesis. CD31 is highly expressed in endothelial cells and the expressions of vascular endothelial growth factor (VEGF) and CD31 have recently been implicated in tumor angiogenesis. HBOT did not change the expression of VEGF but significantly increased the expression of CD31 after 14 days and 28 days of HBOT (*P<0.05)(Figure 4, Table 1). 
Table 1

Semi-quantitative expressions of tumor hypoxia, CD31 and VEGF in groups of mice after 14 and 28 days of HBOT.

\begin{tabular}{|c|c|c|c|c|}
\hline & $\begin{array}{l}\text { Control-14 } \\
(\mathrm{N}=10)\end{array}$ & $\begin{array}{c}\mathrm{HBO}_{2}-14 \\
(\mathrm{~N}=10)\end{array}$ & $\begin{array}{l}\text { Control -28 } \\
(\mathrm{N}=10)\end{array}$ & $\begin{array}{c}\mathrm{HBO}_{2}-28 \\
(\mathrm{~N}=10)\end{array}$ \\
\hline $\begin{array}{l}\text { Tumor hypoxia } \\
\text { (mean } \pm \text { SD) }\end{array}$ & $3.40 \pm 0.55$ & $\star 1.25 \pm 0.96$ & $3.20 \pm 0.37$ & $\star 2.00 \pm 0.01$ \\
\hline $\begin{array}{l}\text { CD31 } \\
(\text { mean } \pm \text { SD })\end{array}$ & $2.20 \pm 0.45$ & $\star 3.25 \pm 0.50$ & $2.00 \pm 0.32$ & $\star 2.25 \pm 0.48$ \\
\hline $\begin{array}{l}\text { VEGF } \\
(\text { mean } \pm S D)\end{array}$ & $1.40 \pm 0.55$ & $1.25 \pm 0.50$ & $1.20 \pm 0.20$ & $1.25 \pm 0.25$ \\
\hline \multirow[t]{5}{*}{ 1. Cell staining } & $0 \%$ & No staining & & 0 \\
\hline & $0-1 \%$ & Nuclear stai & ess than $1 \%$ of cells & 1 \\
\hline & $1-10 \%$ & In $1-10 \%$ of & & 2 \\
\hline & $10-50 \%$ & In $10-50 \%$ of & & 3 \\
\hline & $>50 \%$ & Greater than & & 4 \\
\hline \multicolumn{5}{|c|}{ 2. Nonparametric Kruskal - Wallis test } \\
\hline 3. ${ }^{*} P<0.05$, con & red with Cor & l group & & \\
\hline
\end{tabular}

\section{HBOT decreased p53 protein and increased HIF-1a protein in A549 cells, not Beas-2B cells.}

We examined the effects of HBOT on A549 cells compared with an immortalized human bronchial epithelial cell line, Beas-2B, followed by the cell-based HBOT protocol (Figure 5A). HBOT suppressed the growth of $A 549$ cells, not Beas-2B cells, in a time-dependent manner as measured in Figure 5B. From western blotting analysis, $p 53$ proteins in $A 549$ cells were first downregulated by HBOT and then rebounded to basal level after 6 hours of HBOT, but not Beas-2B cells (Figure $5 C$ ). There's no significant difference in the expression of p53 mRNA after HBOT between $A 549$ and Beas-2B cells (Figure 5D). The $\angle C 3 B I I / I$ ratio was increased by the HBOT and then declined to basal level after HBOT in Beas-2B cells (Figure 5C).

\section{HBOT-reducedp53 protein could be rescued by a proteasome degradation inhibitor, but not an autophagy}




\section{inhibitor in A549 cells.}

The downregulation of $p 53$ protein of $A 549$ cells upon HBOT was consistent at a continuous 3-day exposure; 553 protein was immediately downregulated at the first moment of HBOT exposure and back to the normal after $20 \mathrm{~h}$ exposure (Figure 6). Hypoxia-inducible factor 1 alpha (HIF-1a) protein expression was significantly decreased by HBOT after exposure at the first interval and rebounded to basal level both in A549 and Beas-2B cells (Figure 6); however, the apoptotic biomarkers of cleaved PARP and caspase 3 remained unchanged in both $A 549$ and Beas-2B cells (Figure 6). There was no obvious increaseofsubG1 phase by flow cytometry analysis (Figure 6). With the cytosolic and nuclear fractions, we found that the effect of HBOT on p53proteinsoccurred in the cytosol and nucleus of A549 cells (Figure 7). We tested whether a proteasome degradation inhibitor, MG132 or the autophagy inhibitor 3-methyladenine (3-MA), could rescue the effect of HBOT on p53 and HIF-1a proteins. Our data showed that MG132 could rescue p53 and HIF-7a proteins in A549- HBOT cells (Figure 7). The effect of MG132 on HIF-1a protein was dependent on cell-context and HBOT. In A549 cells, MG132 not only stabilized basal HIF-1a protein, but also further enhanced HIF-1a protein in A549-HBOT cells. However, the HIF-1a protein in MG132-treated Beas-2 cells was still decreased under HBOT condition. 3-MA could suppress the induction of autophagy by HBOT in Beas- $2 B$ cells, suggesting that the autophagy did not affect p53 protein stability in A549 cells by HBOT (Figure 7).

\section{Discussion}

In this study, HBO was used to treat mice transferred with A549 human lung carcinoma in the manner of 2.5 ATA/90 min daily for 2 weeks after 45 days of tumor establishment. HBOT in a cycle of 14 and 28 days both showed significant improvement for tumor hypoxia environment and suppressed tumor growth compared with control mice. Interestingly, tumor vascularity detected by the expressions of CD31 was significantly increased after 14 and 28 days of HBOT; however, the expression of VEGF did not change as measured in semi-quantitative IHC staining analysis. This finding indicated that the improvement of cancer hypoxia and cancer vascularity by HBOT did not promote cancer growth. For tumor angiogenesis, the new blood vessels are formed when the basement membrane of existing blood vessels is broken and while the vascular basement membrane has been breached, endothelial cells will be divided to form vessels and start to grow. VEGF appears to be the first to induce endothelial cell mitosis in tumor angiogenesis [21, 22]. In contrast, CD31, which is a mitogenic factor in wound healing, reacts more sensitive to oxygen tension [23]. HBOT significantly increased the expression of CD31 after 14 days of HBOT. Meanwhile, the volume of A549 tumor cells was not growing as increasing expressions of CD31. To evaluate the direct effect of HBOT in A549 cells, the decrease of p53 and HIF-1a proteins was involved in the ubiquitin-dependent proteasome degradation pathway via the proteasome degradation inhibitor MG132 in A549 cells. The extra effect of MG132 on p53 and HIF-1a proteins were observed by HBOT in A549 cells. In a normal lung epithelial cell line, we also observed the decrease of HIF-1a protein with the HBOT, even in the presence of MG132. The involvement of autophagy was verified via the increasing LC3BII/I ratio and its specific inhibitor, 3-MA, in Beas-2 cells, but not in A549 cells. Our findings suggest 
that HBOT induced tumor suppression in A549-tumor transferred SCID mice might be through modification of tumor microenvironment rather than induction of autophagy in tumor cells themselves.

Malignant tumors have been recognized as a relative contraindication to HBOT. It was concerned that HBOT might have cancer growth-enhancing effects. Our findings indicated that the improvement of tumor hypoxia and tumor vascularity by HBOT did not promote tumor growth but inhibit tumor development, which provided evidence for the potential use of HBOT on tumor malignancy. Although the mechanism of HBOT on tumor suppression is not clear, our experiments argue that HBOT results in tumor apoptosis. Moreover, HBOT can increase the formation of reactive oxygen species (ROS) and induce apoptosis of tumor cells $[24,25]$. In our previous experiments, primary cells and transformed tumor cell lines were treated with either 2.5 ATA or 3.5ATA of $100 \%$ oxygen for $6 \mathrm{~h}$, and a significant percentage of apoptosis was compared. HBO-induced apoptosis of cancer cells was through the intracellular accumulation of $\mathrm{H}_{2} \mathrm{O}_{2}$ and $\mathrm{O}_{2}{ }^{-}$as well as the involvement of phosphorylation of p38 MAPK [17]. In the present study, non-hematopoietic A549-transferred SCID mice were treated with HBO2.5 ATA/90 min daily for 2 weeks and immunoassaying of cleaved-caspase- 3 was demonstrated after 14 and 28 days of HBOT compared with control mice. However, there is no significant difference of apoptotic biomarkers, including cleaved PARP and caspase 3, directly in A549 cells after HBOT. Our current study is consistent with our previous reports that different origins of different cancer cells result in different threshold to HBOTinduced cancer apoptosis. The possible proposal of tumor suppression in HBOT treated murine xenograft model is that HBOT may provide better tissue vascluogenesis rather than angiogenesis to recruit more inflammatory cells in tumor microenvironment. However, the detailed working mechanisms should be addressed in the future studies.

Lung cancers usually start in the cells lining the bronchi, bronchioles or alveoli [26]. The p53 tumor suppressor gene has involved in several stages of lung cancer development [27-30]. It is an important genome protector, and translocates to the nucleus upon cellular stress. Furthermore, wild-type p53 might induce senescence in neighboring tumor cells, and inhibit angiogenesis [31, 32]. Herein, our study demonstrated that HBOT immediately downregulated the endogenous p53 protein, and rebounded to the basal level after 20 hours of treatment; which could provide the help to improve tumor vascularity, but could not directly cause further apoptosis and cell cycle arrest in the cell base analysis. We are the first to examine the effect of HBOT on wild type p53 in A549 cells. Other interesting findings were the levels of CD31 and VEGF expressions upon HBOT. There was no significant change of VEGF expressions after HBOT supporting our hypothesis that HBOT could not further accelerate tumor angiogenesis and lead to tumor metastasis. By contrast, CD31, Platelet endothelial cell adhesion molecule (PECAM-1) was significantly upregulated after HBOT; which might further recruit more immune cells to the microenvironment for immune clearance [33,34]. In addition to p53 protein, HBOT could decrease the HIF-1a protein in A549 and Beas-2 cells, which supported the hypothesis that HBOT could ameliorate the hypoxic condition in cancer cells and prevent metastasis $[35,36]$. Combined with our in vivo and in vitro findings suggest that tumor microenvironment interacting with tumor cells is significantly impacted after 
HBOT in lung cancer. However, the detailed regulatory network of HBOT will be addressed in future studies.

In conclusion, our results demonstrated that HBOT could improve tumor vascularity, tumor hypoxia and potentially target apoptosis-related genes leading to tumor suppression for lung cancer cells. Our present work not only provides direct beneficial evidence for HBOT on lung cancer but also lightens a hope to other solid tumor cancer therapy

\section{Materials And Methods \\ Cell culture}

Human lung carcinoma A549 cell line and an immortalized human bronchial epithelial cell line, Beas-2B, were purchased from the Food Industry Research and Development Institute (Hsinchu, Taiwan, R.O.C.). The cells were cultured at $10^{6}$ cells $/ \mathrm{ml}$ in RPMI 1640 with $10 \%$ fetal calf serum, $2 \mathrm{mM}$ L-glutamine, $1 \mathrm{mM}$ sodium pyruvate, $4.5 \mathrm{~g} / \mathrm{I}$ glucose, $10 \mathrm{mM}$ HEPES, and $1.5 \mathrm{~g} / \mathrm{I}$ sodium bicarbonate. Individual cultures were maintained for no more than 2 months.

\section{Mice}

50 of C.B-17 SCID female mice were purchased from National Taiwan Medical Animal Center and maintained in pathogen-free, individually ventilated cage sat National Defense Medical CenterLaboratory Animal Center (NDMCLAC), Taiwan. Routinely sterilized, rodent diets (Meika) were used to feed the mice. The mice were used for the experiments at the ages of 6 to $8 \mathrm{wk}$. All animal experiments were in accordance with NDMCLAC guidelines of IACUC. All animal protocols were approved by the IACUC in Taiwan (IACUC-101A01-002, IACUC-13-264). The study was carried out in compliance with the ARRIVE guidelines.

\section{Injection of A549 tumors}

Mice were anesthetized by intra-peritoneum (i.p.) injection of pentobarbital: $40 \mathrm{mg} / \mathrm{kg}$ body weight. The abdomen was sheared and sterilized with 2-isopropanol (70\%).A549 cells were diluted to $6 \times 10^{6}$ cells in a volume of $0.2 \mathrm{ml}$ RPMI 1640 and transferred to each recipient at the neck site by subcutaneous injection. The diameter of tumor mass was monitored two times per week and only mice with tumor volume larger than $500 \mathrm{~mm}^{3}$ were selected for further experiments.

\section{Hyperbaric oxygen treatment (HBOT)}


A549-tumor transferred SCID mice were either non-exposed or exposed to HBO in a hyperbaric animal chamber ( $98 \% \mathrm{O}_{2}, 2 \% \mathrm{CO}_{2}$ at $2.5 \mathrm{ATA} ; 0.5 \mathrm{ATA} / \mathrm{min}$ to a pressure of $2.5 \mathrm{ATA}$ ) (Longshin Gas Ltd. Taipei, Taiwan, ROC)for 90 min once a day over two weeks beginning at the $45^{\text {th }}$ date after tumor injection. Cells in groups were exposed to HBOT (98\% 02, 2\% CO2 at 3.5 ATA ) (Longshin Gas Ltd., Taipei, Taiwan, ROC)in a hyperbaric chamber for $90 \mathrm{~min}$. Control cultures for each experiment were placed in an incubator at $37 \circ \mathrm{C}, 21 \% 02,5 \% \mathrm{CO} 2$ at 1 ATA. This hyperbaric chamber was maintained at a concentration of $95 \%$ oxygen, and $\mathrm{CO}_{2}$ was exhausted at a rate of $10-12 \mathrm{~L} / \mathrm{min}$. Reaching the end of the treatment, the chamber was slowly decompressed at $0.5 \mathrm{ATA} / \mathrm{min}$ pressure.

\section{Tumor volume}

We measured tumor volume two times in a week and calculated the tumor size as length $(\mathrm{L}) \mathrm{x}$ width $(\mathrm{W}) \mathrm{x}$ height $(\mathrm{H})$.

\section{Detection of tumor hypoxia}

Hypoxyprobe ${ }^{\mathrm{TM}}$-1 kit (Pimonidazole Hydrochloride) (Chemicon International) was used to measure tissue hypoxia in solid tumors. A dose of $60 \mathrm{mg} / \mathrm{kg}$ bodyweight of Hypoxyprobe $\mathrm{TM}^{\mathrm{TM}} 1$ kit was injected intraperitoneally to A549-transferred SCID mice and mice were sacrificed after $1 \mathrm{~h}$. Tumor mass was removed and embedded as OCT/liquid nitrogen cryostat section.

\section{Tumor histopathology}

Tumor cells were removed from mice for either frozen or paraffin sections. For frozen sections, tissue cells were snap-frozen in dry ice-cold 2-methylbutane and embedded in TISSUE-TEC (Miles, Elkhart, IN, USA). Freshly cut sections ( $5 \mathrm{~mm}$ ) were mounted on clean glass slides coated with poly-L-lysine (Sigma Chemical Co, USA). They were further rapidly air-dried and stored at $-80^{\circ} \mathrm{C}$ until used for immunohistochemical staining. The fixed sections were incubated with normal goat serum diluted 1:5 in PBS for $15 \mathrm{~min}$ at room temperature to block nonspecific staining. Sections were later incubated with goat anti-mouse IgG primary antibody (anti-CD31; BD Pharmingen, San Diego, CA, USA)in PBS for $1 \mathrm{~h}$ at room temperature and washed with PBS for 5 min with gentle shaking. After washing in PBS, they were interacted with secondary antibodies and developed with DAKO Kit-DAB chromogen for 6 min. The slides were washed with $\mathrm{Q}$ water and incubated with hemataxylin for the staining of the nucleus. The sections were immersed with 75\%-95\%-100\% alcohol and xylene before covered with a coverslip. The processing, embedding and sectioning of paraffin blocks were performed in Dr. Koichi Tsuneyama's pathology laboratory and the paraffin sections were deparaffinization and re-hydration before immunohistochemical staining. 


\section{Western blotting}

Equivalent amounts of protein were mixed with an equal volume of 2x Laemmli buffer, boiled and resolved on $10 \%$ SDS-PAGE gels. After the protein was transferred to nitrocellulose membranes by electroblotting (Bio-Rad, Richmond, CA, USA), the blots were blocked by overnight incubation with $5 \%$ nonfat dry milk in Tris/boric acid/sodium chloride/Tween 20 and subsequently probed with antibodies againstp53, ACTN, and Nrf-2 (Santa Cruz, USA), LC3B,HIF-1a,PARP,Caspase-3, and p-p53 (Ser15) (Cell Signaling, USA), and g-H2A.x (Abcam, UK). Immunoreactive proteins were visualized using horseradish peroxidase-linked secondary antibodies and further with ECL (Enhanced-chemiluminescence western blotting kit) (Amersham Biosciences, Pittsburgh, PA, U.S.A).

\section{Reverse transcription-polymerase chain reaction (RT-PCR)}

Total RNA was isolated using TRIzol (Thermo Fisher Scientific, USA) reagent according to the manufacturer's instructions. For 1st-strand cDNA synthesis, $1 \mu \mathrm{g}$ of total RNA was reverse transcribed using $200 \mathrm{U}$ of M-MLV reverse transcriptase for $60 \mathrm{~min}$ at $37^{\circ} \mathrm{C}$ (Epicentre Biotechnologies, USA). Specific primers (for p53: Forward: 5'-CTCTGACTGTACCACCATCCACTA-3'; Reverse: 5'-

GAGTTCCAAGGCCTCATTCAGCTC-3'; for GAPDH: Forward: 5'-CTT CAT TGA CCT CAA CTA C-3'; Reverse: 5'-GCC ATC CAC AGT CTT CTG-3'), dNTP, and Taq DNA polymerase were added for subsequent PCR reactions, which were processed using a Veriti ${ }^{\top \mathrm{M}}$ Simpli Thermal Cycler (Applied Biosystems, CA, USA).

\section{Statistical analysis}

Unpaired t-test, one-way ANOVA and Nonparametric Kruskal-Wallis test were used to compare differences between means of groups and statistical analysis was performed using SPSS. One-Dscan analysis software was used to analyze the expression contents of proteins in western blotting.

\section{Declarations}

\section{Authors' contributions}

SY Chen, MH Yen and SM Huang designed the research. K Tsuneyama and SM Huang supported the experiments. JT Lee and JL Chen collected and analyzed the data. All authors interpreted the results and approved the manuscript.

\section{Ethical Approval and Consent to participate}

All animal experiments were in accordance with the Laboratory Animal Center of National Defense Medical Center (NDMCLAC) guidelines of IACUC. All animal protocols were approved by the IACUC in 


\section{Funding}

This study was supported in part by a grant-in-aid from the National Science Council (NSC 93-2314-B016-026), Tri-Service General Hospital (TSGH) Foundation (TSGH-C94-46), Cardinal Tien Hospital (CTH106A-1-2A01, CTH-106A-2C02, CTH108A-2A03), and grant CH-NDMC-108-02 from Cheng Hsin General Hospital

\section{References}

1. Kizaka-Kondoh, S. Inoue, M. Harada, H. Hiraoka, M. Tumor hypoxia: A target for selective cancer therapy. Cancer Sci. 94(12), 1021-8 (2003).

2. Jing, X. et al Role of Hypoxia in Cancer Therapy by Regulating the Tumor Microenvironment. Mol Cancer. 18(1), 157 (2019).

3. Wu, T. Dai, Y. Tumor microenvironment and therapeutic response. Cancer Lett. 387, 61 (2017).

4. Brown, JM. Giaccia, AJ. The unique physiology of solid tumors: opportunities (and problems) for cancer therapy. Cancer Res. 58, 1408-1416 (1998).

5. Carmeliet, P. et al. Role of HIF-1alpha in hypoxia-mediated apoptosis, cell proliferation and tumour angiogenesis. Nature. 394(6692), 485-90 (1998).

6. Moulder, JE. Rockwell, S. Tumor hypoxia: its impact on cancer therapy. Cancer Metastasis Rev. 5(4), 313-41 (1987)..

7. Mizuguchi, T. et al. Hyperbaric oxygen stimulates cell proliferation and normalizes multidrug resistance protein-2 protein localization in primary rat hepatocytes. Wound Repair Regen. 13(6), 5517 (2005).

8. Kang, TS. Gorti, GK. Quan, SY. Ho, M. Koch, RJ. Effect of hyperbaric oxygen on the growth factor profile of fibroblasts. Arch Facial Plast Surg. 6(1), 31-5 (2004).

9. Feldmeier, J. Carl, U. Hartmann, K. Sminia, P. Hyperbaric oxygen: does it promote growth or recurrence of malignancy? Undersea Hyperb Med. 30(1), 1-18 (2003).

10. Ashamalla, HL. Thom, SR. Goldwein, JW. Hyperbaric oxygen therapy for the treatment of radiationinduced sequelae in children. The University of Pennsylvania experience. Cancer. 77(11), 2407-12 (1996).

11. Stępień, K. Ostrowski, RP. Matyja, E. Hyperbaric oxygen as an adjunctive therapy in treatment of malignancies, including brain tumours. Med Oncol. 33(9), 101 (2016)

12. Graham, K. Unger, E. Overcoming tumor hypoxia as a barrier to radiotherapy, chemotherapy and immunotherapy in cancertreatment. Int J Nanomedicine. 13, 6049-6058 (2018).

13. Crowther, M. Brown, NJ. Bishop, ET. Lewis, CE. Microenvironmental influence on macrophage regulation of angiogenesis in wounds and malignant tumors. J Leukoc Biol. 70(4), 478-90 (2001). 
14. Liu, S. Tian, Y. Chlenski, A. Yang, Q. Salwen, HR. Cohn, SL. 'Cross-talk' between Schwannian stroma and neuroblasts promotes neuroblastoma tumor differentiation and inhibits angiogenesis. Cancer Lett. 228(1-2), 125-31 (2005).

15. Quail, DF. Joyce, JA. Microenvironmental regulation of tumor progression and metastasis. Nat Med. 19(11), 1423-37 (2013).

16. Chen, SY. et al. Early hyperbaric oxygen therapy attenuates disease severity in lupus-prone autoimmune (NZB x NZW) F1 mice. Clin Immunol. 108(2), 103-10 (2003).

17. Chen, YC. et al. Apoptosis of T-leukemia and B-myeloma cancer cells induced by hyperbaric oxygen increased phosphorylation of p38 MAPK. Leuk Res. 31(6), 805-15 (2007).

18. Wang, Y. et al. Hyperbaricoxygen rescues lungcancercells from chemical hypoxia-induced low differentiation and apoptosis resistance. Exp Lung Res. 44(8-9), 417-423 (2018).

19. Kawasoe, Y. Yokouchi, M. Ueno, Y. Iwaya, H. Yoshida, H. Komiya, S. Hyperbaricoxygenas a chemotherapy adjuvant in the treatment of osteosarcoma. Oncol Rep. 22(5), 1045-50 (2009).

20. Koukourakis, MI. Giatromanolaki, A. Sivridis, E. Fezoulidis, I. Cancer Vascularization: Implications in Radiotherapy? Int J Radiat Oncol Biol Phys. 48(2), 545-53 (2000).

21. Ferrara, N. Gerber, HP, LeCouter, J. The biology of VEGF and its receptors. Nat Med. 9(6), 669-76 (2003).

22. Rak, J et al. Oncogenes and tumor angiogenesis: differential modes of vascular endothelial growth factor up-regulation in ras-transformed epithelial cells and fibroblasts. Cancer Res. 60(2), 490-8 (2000).

23. DeLisser, HM. et al. Involvement of endothelial PECAM-1/CD31 in angiogenesis. Am J Pathol. 151(3), 671-7 (1997).

24. Matsunami, T. et al. Enhancement of reactiveoxygenspecies and induction of apoptosisin streptozotocin-induced diabetic rats under hyperbaricoxygenexposure. Int J. Clin Exp Pathol. 4(3), 255-66 (2011).

25. Weidinger, A. Kozlov, AV. Biological activities of reactive oxygen and nitrogen species: oxidative stress versus signal transduction. Biomolecules. 5, 472-484 (2015).

26. Zito Marino, F.et al. Molecular heterogeneity in lung cancer: from mechanisms of origin to clinical implications.Int J Med Sci. 16(7), 981-989 (2019).

27. Brambilla, E. et al. p53 mutant immunophenotype and deregulation of p53 transcription pathway (Bcl2, Bax, and Waf1) in precursor bronchial lesions of lung cancer. Clin Cancer Res.4, 1609-1618 (1998).

28. Hirano, T. Franzen, B. Kato, H. Ebihara, Y. Auer, G. Genesis of squamous cell lung carcinoma. Sequential changes of proliferation, DNA ploidy, and p53 expression. Am J Pathol.144, 296-302 (1994).

29. Li, ZH. Zheng, J. Weiss, LM. Shibata, D. c-k-ras and p53 mutations occur very early in adenocarcinoma of the lung. Am J Pathol.144, 303-309 (1994). 
30. Xu, Y. et al. Mutated p53 Promotes the Symmetric Self-Renewal of Cisplatin-Resistant Lung Cancer Stem-Like Cells and Inhibits the Recruitment of Macrophages. J Immunol Res.747853810.1155/7478538 (2019).

31. Xu, J.et al. Differential Radiation Sensitivity in p53Wild-Type and p53-Deficient Tumor Cells Associated with Senescencebut not Apoptosis or (Nonprotective) Autophagy.Radiat Res. 190(5), 538557 (2018).

32. Vilgelm, AE.et al.Mdm2 and aurora kinase a inhibitors synergize to block melanoma growth by driving apoptosis and immuneclearanceof tumor cells.Cancer Res. 75(1), 181-93 (2015).

33. Woodfin, A. Voisin, MB. Nourshargh, S.PECAM-1: a multi-functional molecule in inflammation and vascular biology.ArteriosclerThrombVasc Biol. 27(12), 2514-23 (2007).

34. Cheung, K. et al. CD31 signals confer immune privilege to the vascular endothelium.Proc Natl Acad Sci U S A. 112(43), E5815-24 (2015).

35. Anguiano-Hernandez, YM. et al. Modification of HIF-1a, NF-aкB, IGFBP-3,VEGF and adiponectin in diabetic foot ulcers treated with hyperbaricoxygen.Undersea Hyperb Med. 46(1), 35-44 (2019).

36. Sunkari, VG. et al. Hyperbaricoxygentherapy activates hypoxia-induciblefactor1(HIF-1), which contributes to improved wound healing in diabetic mice. Wound Repair Regen. 23(1), 98-103 (2015).

\section{Figures}

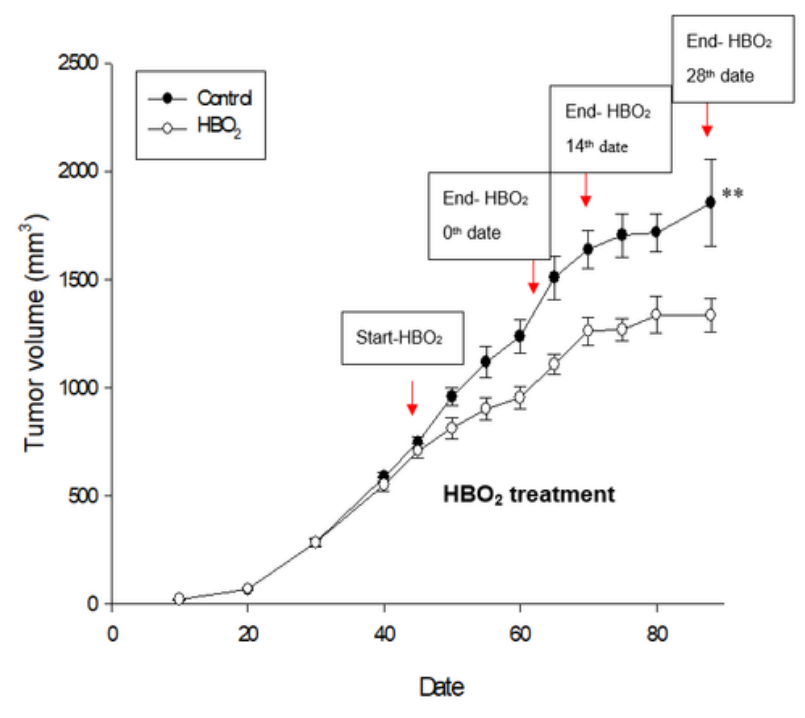

A

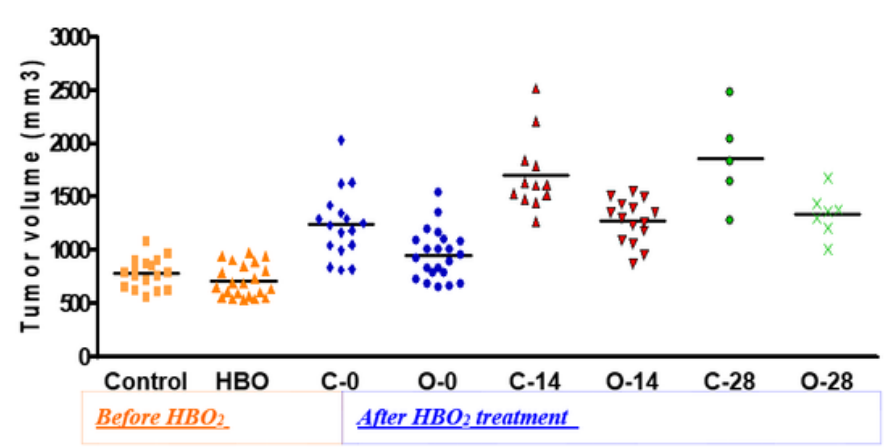

B

\section{Figure 1}

A549-tumor transferred SCID mice were either non-exposed or exposed to HBO2(98\% 02, 2\% CO2 at 2.5 ATAfor2.5 ATA 90 min 5 times in a week beginning at the 45th date after tumor injection and were monitored for the tumor volume twice in a week. Tumor volume was calculated as length $(L) x$ width (W) $x$ height $(H)$. (A) The growth curve of A549 cells in SCID mice. Only the mice with tumor volume larger 
than $500 \mathrm{~mm} 3$ were selected (B) The comparison of tumor volume among groups of mice before or after HBOT. For each dot-point represents the tumor volume of one A549-tumor transferred SCID mice. HBOT effectively suppressed tumor growth after 14 days $(* * P<0.01)$ and 28 days $\left({ }^{*} P<0.01\right)$ of tumor transfer. $\triangle \mathrm{C}-0$ represents for control mice at the first date comparing with HBOT mice; 0-0 represents for mice receiving the first date of $\mathrm{HBOT}$; $\mathrm{C}-14$ days represents for control mice after 14 days comparing with HBOT mice; 0-14 days represents for mice after 14 days of HBOT; C-28 days represents for control mice

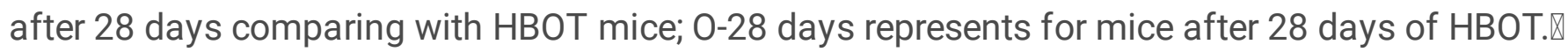

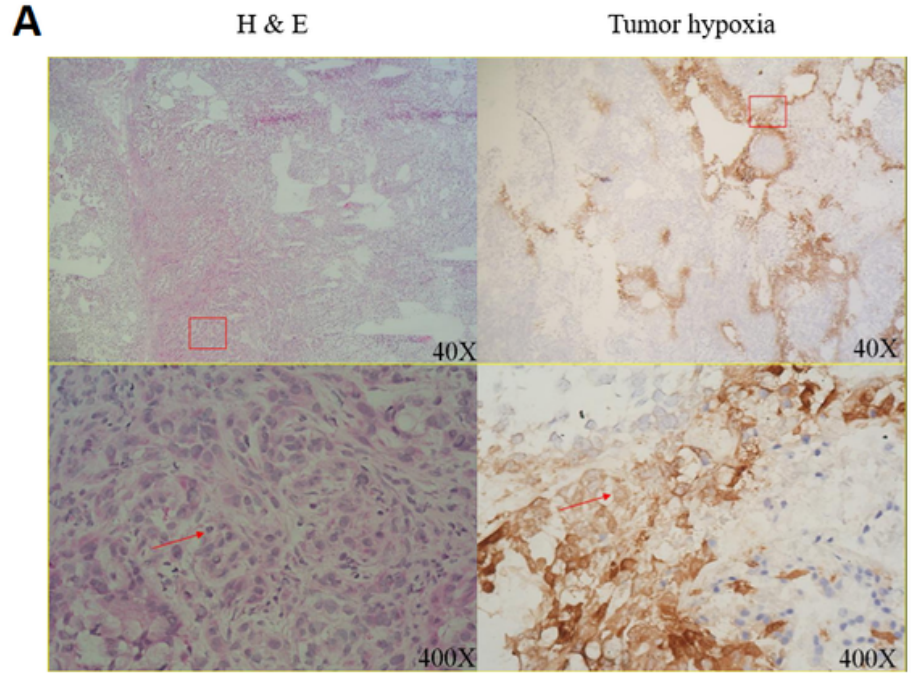

B
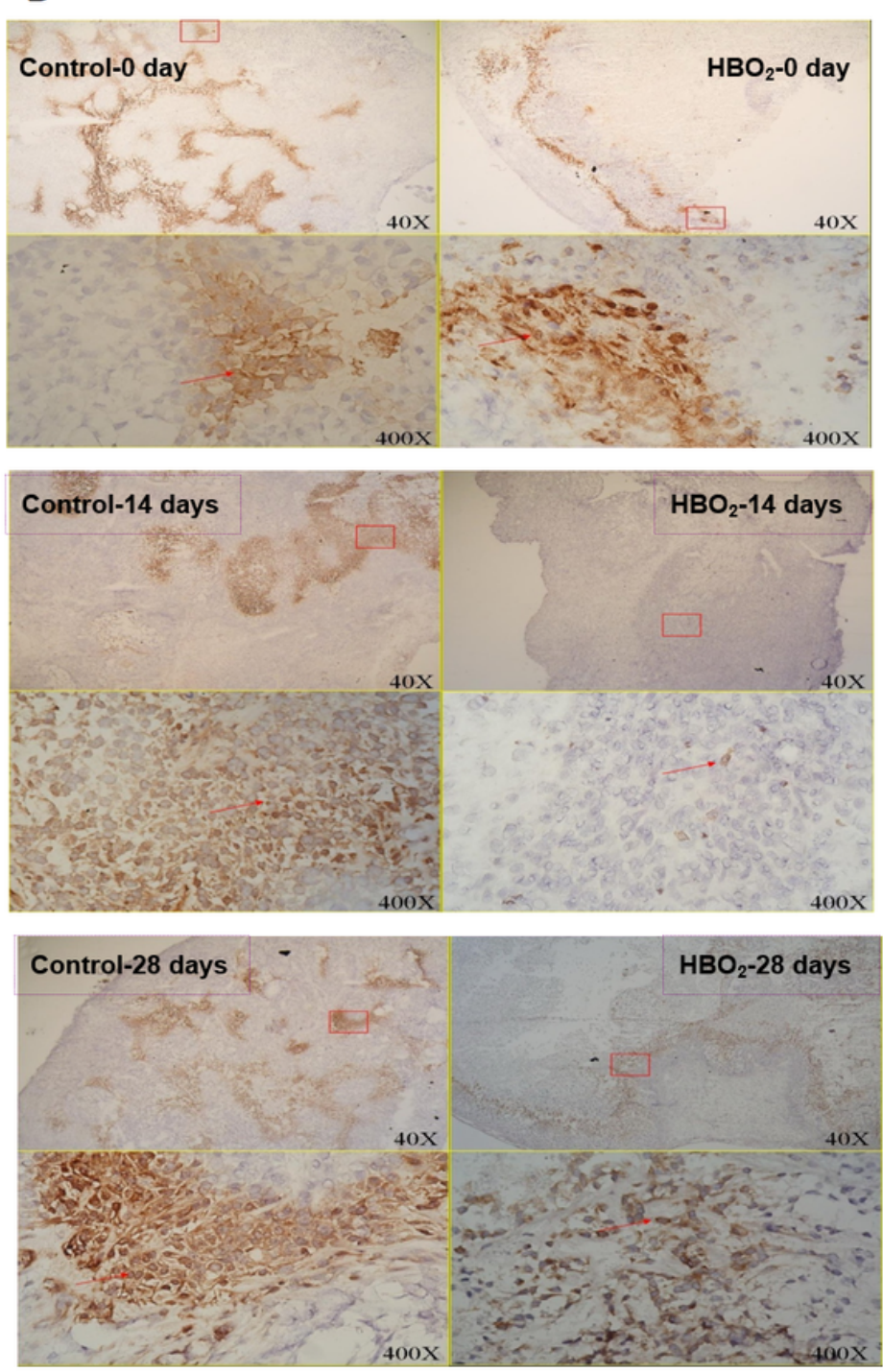

\section{Figure 2}

Immunohistochemical staining of tumor sections from A549- transferred SCID mice. (A) Haematoxylin \& Eosin (H \& E) staining and immunostaining of HypoxyprobeTM-1 probe of A549 tumor frozen sections after 45 days of tumor transfer.(H \& E: $\mathrm{H}$ and E staining; tumor hypoxia: Immunostaining of HypoxyprobeTM-1 probe) (B) Comparisons of tumor hypoxia in groups of mice after 14 and 28 days of HBOT. (Control-0: control mice at the first date comparing with HBOT mice; HBO2-0: mice receiving the 
first date of HBOT; Control-14: control mice after 14 days comparing with HBOT mice; HBO2-14: mice after 14 days of HBOT; Control-28: control mice after 28 days comparing with HBOT mice; HBO2-28: mice after 28 days of HBOT.)
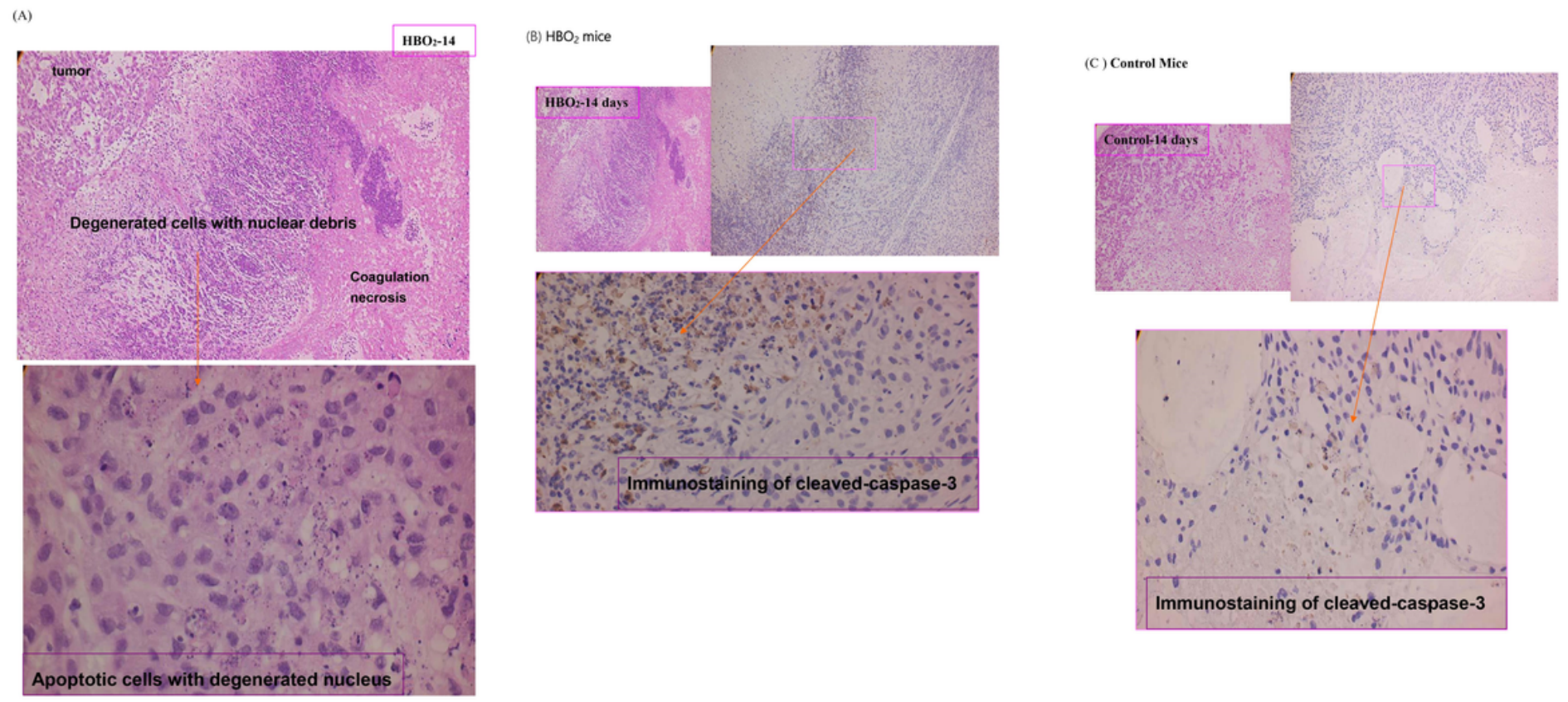

\section{Figure 3}

Immunohistochemistry staining of tumor sections from A549- transferred SCID mice. (A) Tumor paraffin sections of HBO2-14th date showed zonal inflammatory cells and degenerated cells aggregation in the border of coagulation necrosis. Immunostaining of cleaved-caspase-3 after 14 days of HBOT in HBO2 group (B) and control group (C). Note that, in HBOT group, many cleaved-caspase-3 positive apoptotic cells were located in the border between coagulation necrosis and viable tumor cells. 

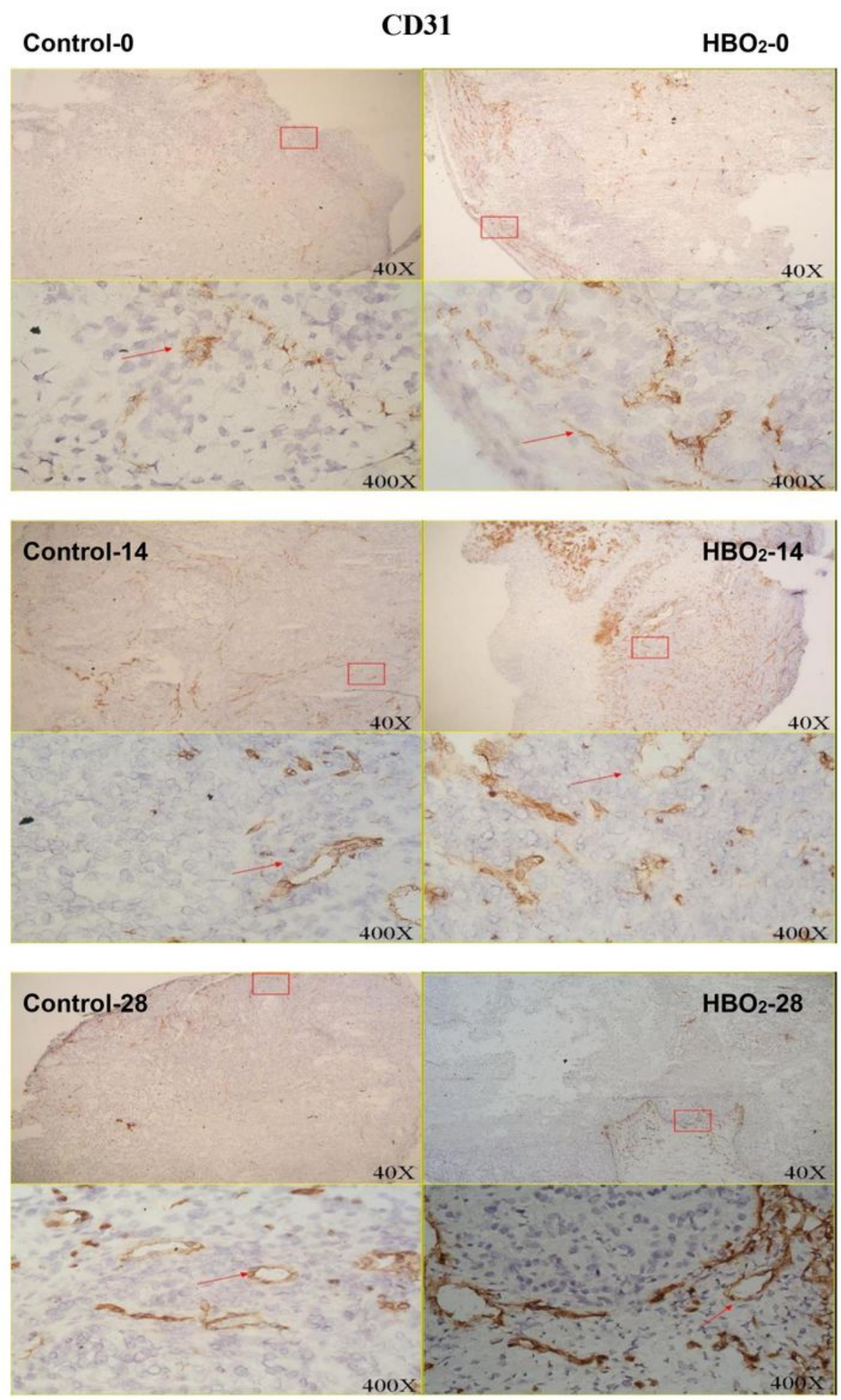

\section{Figure 4}

Comparative analysis of immunohistochemical CD31 antigen expressions from groups of mice. Significantly increase of CD31 was observed after 14 days and 28 days of HBOT exposure 


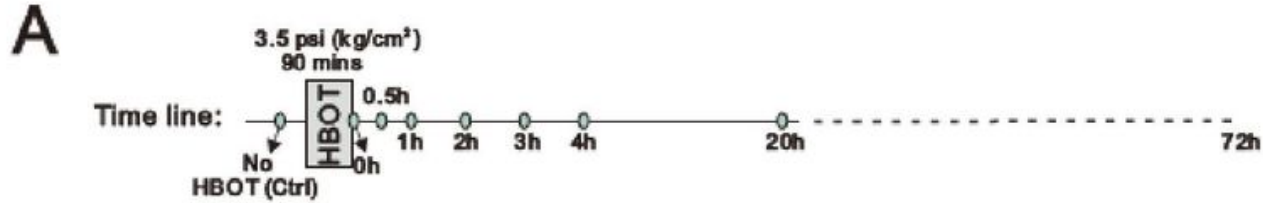

B

A549

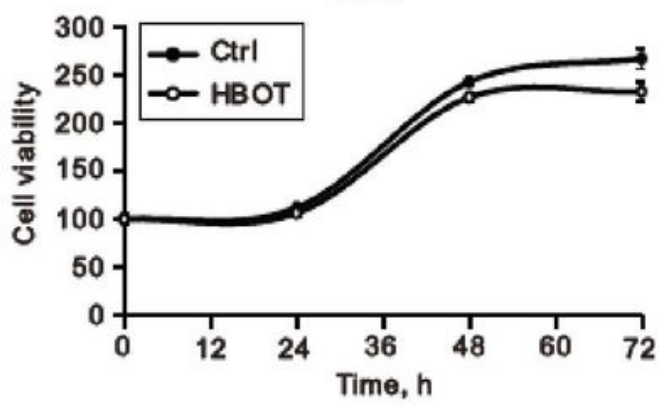

C

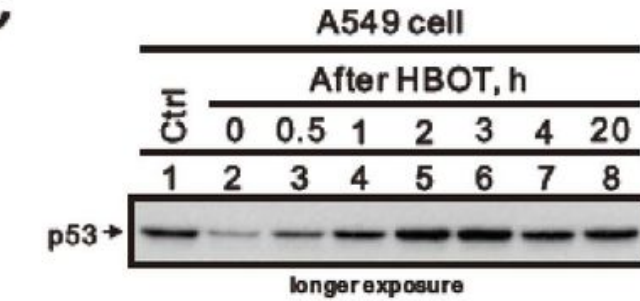

p53

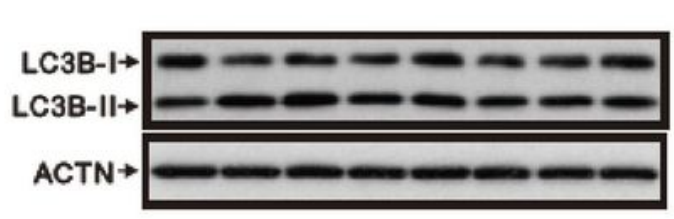

D

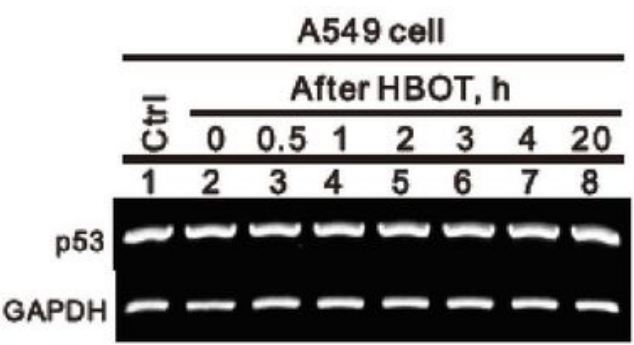

Beas-2B

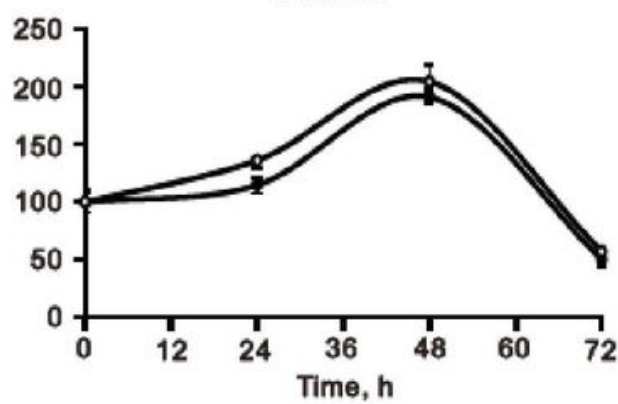

Beas-2B cell

\begin{tabular}{|c|c|c|c|c|c|c|c|}
\hline \multirow{2}{*}{ 돈 } & \multicolumn{7}{|c|}{ After HBOT, h } \\
\hline & 0 & 0.5 & 1 & 2 & 3 & 4 & 20 \\
\hline 1 & 2 & 3 & 4 & 5 & 6 & 7 & 8 \\
\hline
\end{tabular}

longer exposure

shorter exposure

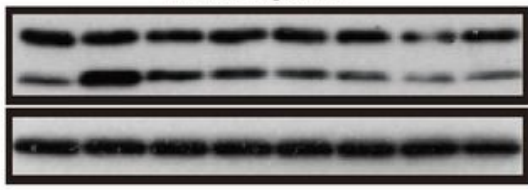

Beas-2B cell

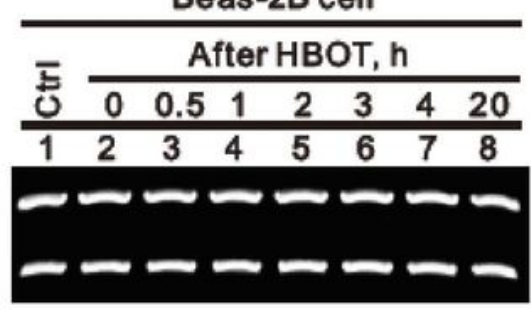

\section{Figure 5}

The HBOT protocol and its transient effect on A549 and Beas-2 cell lines. (A) The HBOT protocol was applied in A549 and Beas-2 cell lines. (B) The cell viability profiles were analyzed in A549 and Beas-2 cell lines. ( $C$ and D) The HBOT A549 and Beas-2 cells were treated with indicated conditions and then cell lysates were subject to the (C) Western analysis for antibodies against p53, LC3B, and control protein ACTN and (D) RT-PCR analysis for p53 mRNA and control GAPDH mRNA. The grouping of gels/blots was 
cropped from different parts of the different gels and the full-length gels and blots were included in the supplementary figure 5. Please note that there was no difference in the expression patterns for both shorter and longer exposure (Supplementary figure 5).

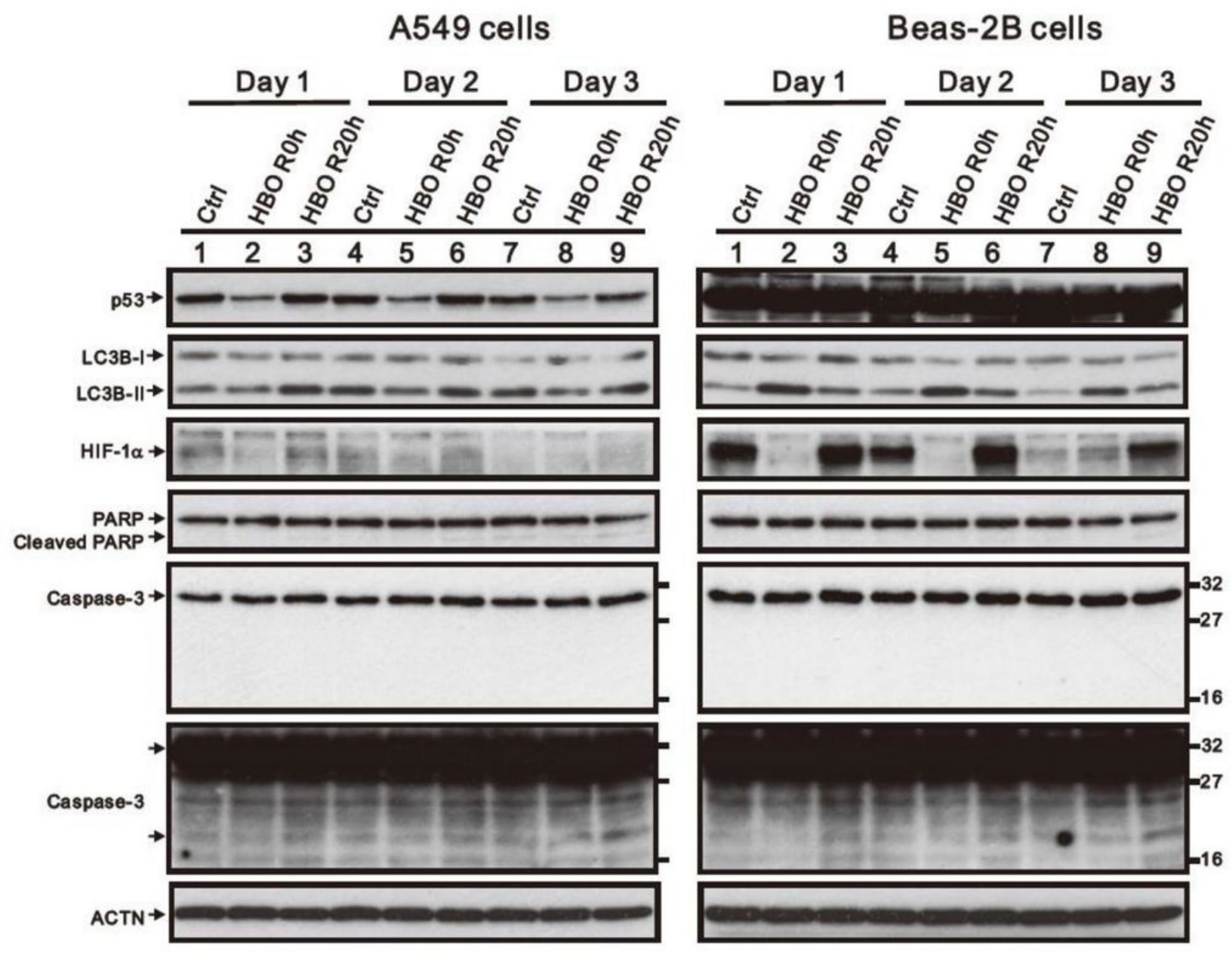

Figure 6

The HBOT effect on stress proteins on A549 and Beas- 2 cell lines. The HBOT A549 and Beas-2 cells were treated with 3.5 ATA for 90 mins and then lysates were subject to the Western analysis for antibodies against p53, LC3B, HIF-1a, PARP, Caspase 3, and control protein ACTN. The grouping of gels/blots was cropped from different parts of the different gels and the full-length gels and blots were included in the supplementary figure 6 . Please note that there was no difference in the expression patterns for both shorter and longer exposure (Supplementary figure 6). 


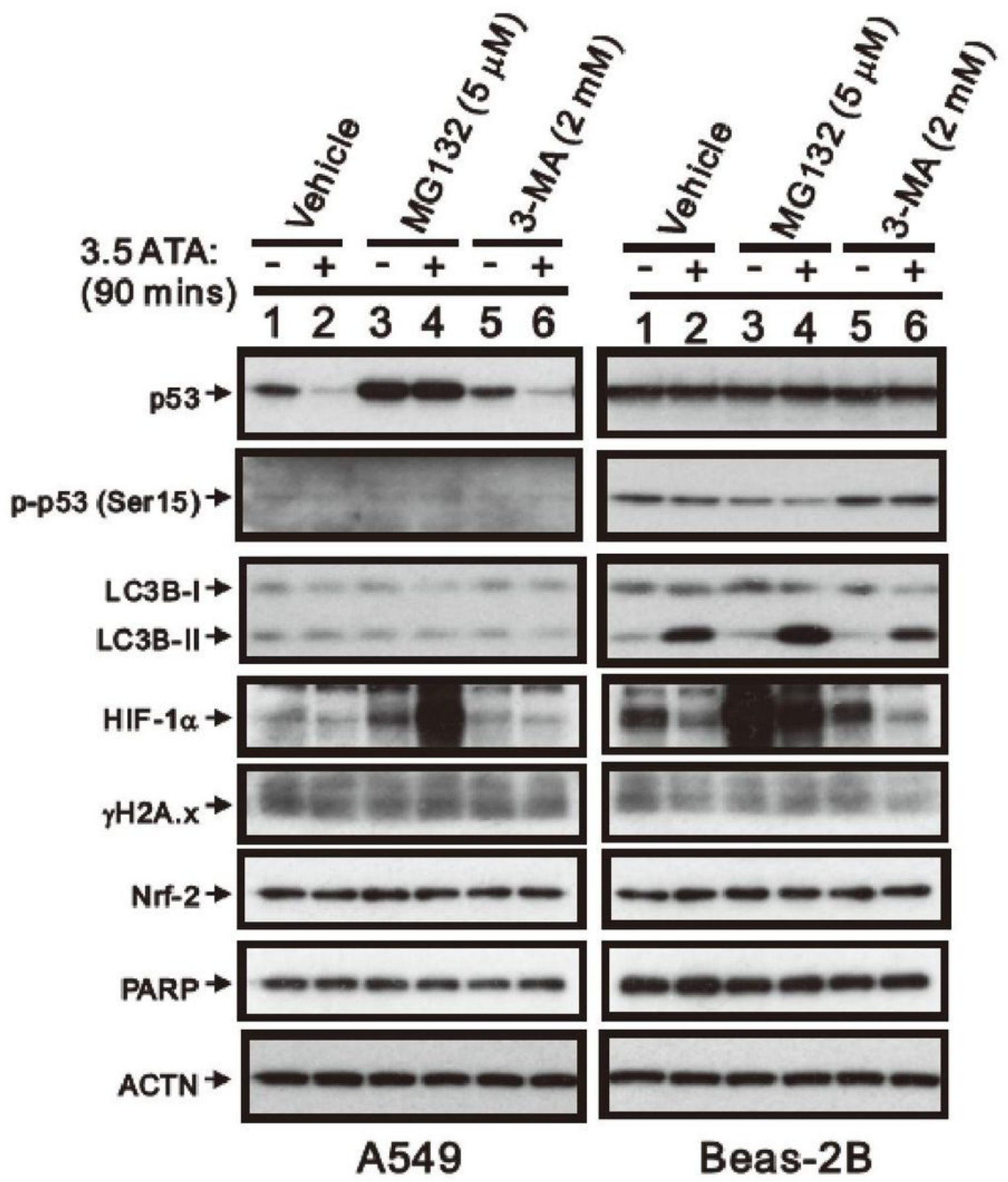

Figure 7

The reduced p53 protein level by HBOT was modulated through a ubiquitin-dependent proteasome degradation in A549 cells. The HBOT A549 and Beas-2 cell were treated with 3.5 ATA for 90 mins in the presence of 5mM MG132 (a proteasome degradation inhibitor) or 2mM 3-MA(an autophagy inhibitor) for $4 \mathrm{hrs}$. These cell lysates were subject to the Western analysis for antibodies against p53, LC3B, HIF-1a, 
yH2A.x, Nrf2, PARP, and control protein ACTN. The grouping of gels/blots was cropped from different parts of the different gels and the full-length gels and blots were included in the supplementary figure 7.

\section{Supplementary Files}

This is a list of supplementary files associated with this preprint. Click to download.

- Supplementaryfigure5.doc

- Supplementaryfigure6.doc

- Supplementaryfigure7.doc 